\title{
The Influence of Psychological Flexibility of Nurses on Occupational Stress during the COVID-19 under the Background of Big Data: The Mediating Effect of Error Management Climate
}

\author{
Bo Lin ${ }^{1}$ Junbo $\mathrm{Ma}^{2}$ Keqiang Chi*3 \\ ${ }^{1}$ Department of Economics and Management Dalian University Dalian, China \\ ${ }^{2}$ Department of Economics and Management Dalian University Dalian, China \\ ${ }^{3}$ Dalian University Dalian, China
}

\begin{abstract}
Objective: Understand the current situation of hospital error management climate, nurses' psychological flexibility and occupational stress during the COVID-19 under the background of big data, to explore the influence of nurses' psychological flexibility on occupational stress and the intermediary role of error management climate. Provide a basis for improving the hospital's error management climate during the COVID-19 and major public health emergencies under the background of big data, provide an improvement path for improving nurses' psychological flexibility and reducing occupational stress level. Methods: In April 2020, 587 nurses were investigated with error management climate scale, psychological flexibility scale and occupational stress scale. Results: Psychological flexibility is negatively correlated with occupational stress, and error management climate mediates the relationship between the two. Conclusion: Different types of nurses perceive different levels of error management climate, psychological flexibility and occupational stress, among them, gender, education background and working years may be the influencing factors; the higher the level of psychological flexibility of nurses; the better the level of perceived error management climate, and the lower the level of occupational stress of nurses.
\end{abstract}

\section{INTRODUCTION}

At 0:00 on May 2, 2020 Beijing time, with the level of public health emergency response in Hubei Province changed from level 1 to level 2, there are no provinces in the mainland of China with level 1 public health emergency response level, all of which have cancelled level 1 response. The outbreak of the novel coronavirus pneumonia (COVID-19) since December 2019 has lasted for more than 4 months and has finally ushered in the dawn of victory. Although the COVID-19 has been basically controlled and mastered, the global situation is still not optimistic. As of May 1, 2020 Beijing time, the People's Daily published news that a total of 3,225,340 cases have been diagnosed in 212 countries and regions, and a total of 232,910 deaths have occurred in new coronary pneumonia worldwide. Therefore, the prevention and control measures for the epidemic should not be relaxed. During the outbreak, nurses stood on the front line of epidemic prevention and rescue, directly dealing with patients with new coronary pneumonia, and faced the risk of being infected by the disease at any time ${ }^{[1]}$. The COVID-19 is highly infectious, and has a wide spread range, despite the fact that nurses possess certain medical skills and first aid ability ${ }^{[2]}$. Therefore, compared with doctors with mature medical standards, nursing staff are still prone to cause various psychological problems when facing major epidemics ${ }^{[3-4]}$. Therefore, this study will explore the status of nurses' occupational stress (OS) and its influencing factors during the COVID-19 under the background of big data.

Psychological flexibility (PF) is a kind of internal adjustment ability, which means that an individual can consciously choose the required behavior under the guidance of a clear personal value according to his own environment and various conditions, and then achieve the goal ${ }^{[5]}$. Relevant research shows that psychological flexibility plays an important role in alleviating various negative emotions and maintaining normal life and social functions ${ }^{[6]}$. At the same time, according to the theory of ecosystem, the development of individuals is affected by the interaction between individuals and the environment, that is, in addition to individual factors, environmental factors also have an important impact on individual behavior. Faced with novel coronavirus pneumonia, nurses are likely to make mistakes in their work. As an important organizational situational factor, error management climate (EMC) emphasizes the attitude of organizations towards errors, specifically, it refers to the organization's attitude, handling process and practice related to or caused by errors ${ }^{[7]}$. Error management climate includes three dimensions: error thinking and learning (ETL), error communication (EC) and error correction 
feedback (ECF).

Based on the above analysis, this study takes nurses as the research object to understand the current situation of hospital error management, nurses' psychological flexibility and occupational stress in the context of the new coronary pneumonia epidemic. To explore the influence of nurses' psychological flexibility on occupational stress and the mediating role of error management climate, provide a basis for improving the hospital's error management climate in the context of the new coronary pneumonia epidemic and major public health emergencies, and provide an improvement path for improving nurses' psychological flexibility and reducing occupational stress level. Figure 1 shows the theoretical model of this study.



Fig 1. Theoretical Model

\section{Objects and methods}

\subsection{Objects}

A simple random sampling method was used to investigate nurses willing to participate in the survey. A total of 660 questionnaires were issued and 660 were recovered. After eliminating the invalid questionnaires, 587 valid questionnaires were finally included in the statistical analysis, with an effective recovery rate of $88.9 \%$.

\subsection{Research Tools}

\subsubsection{Psychological Flexibility Scale.}

The second version of the acceptance and action questionnaire was adopted by Cao Jing et al. ${ }^{[8]}$. There are 7 items in the scale. In this study, the method of Hulbert Williams et al. was used to score reversely ${ }^{[9]}$, and the sum of 7 items represents the level of psychological flexibility. In this study, Likert 5-point scoring method was used. The higher the total score, the lower the degree of empirical avoidance and the higher the level of psychological flexibility. The Cronbach 's $\alpha$ coefficient of the scale in this study was 0.887 .

\subsubsection{Error Management Climate Scale.}

This study draws on the error management climate scale of Dyck, Frese and He ${ }^{[10-11]}$. There are 12 items in the scale, including three dimensions of error thinking and learning, error communication and error correction feedback. In this study, Likert 5-point scoring method was used to score, the higher the total score, the higher the degree of openness of the error management climate. In this study, the Cronbach 's $\alpha$ coefficient of the scale is 0.940, and the Cronbach 's $\alpha$ coefficients of three dimensions of thinking and learning, error communication and error correction feedback are $0.899,0.881$, and 0.818 , respectively.

\subsubsection{Occupational Stress Scale.}

Using Cao's work pressure source scale ${ }^{[12]}$. The scale has a total of 5 items and is scored using Likert 5-level scoring. The higher the total score, the greater the perceived work pressure. The Cronbach 's $\alpha$ coefficient of this scale in this study was 0.757 .

\subsubsection{General Investigation.}

The main content includes: basic personal information such as gender, working years, and the number of night shifts per month.

\subsection{Statistical Methods}

Using software such as SPSS20.0, first, use the reliability analysis to test the reliability of the questionnaires recovered; Secondly, use analysis of variance, $t$ test, etc. to analyze the scores of psychological flexibility, error management climate and occupational stress under different conditions; Finally, we use correlation factor analysis and regression analysis to explore the intermediary role of error management climate between psychological flexibility and occupational stress. $\mathrm{P}<0.05$ indicates that there is significant difference in statistical results.

\section{Results}

\subsection{Survey of the Basic Situation}

Table1. General Information of Respondents $(n=587)$

\begin{tabular}{|c|c|c|}
\hline Projects & $\begin{array}{c}\text { Number of } \\
\text { cases }\end{array}$ & Proportion(\%) \\
\hline \multicolumn{3}{|l|}{ Gender } \\
\hline Male & 62 & 10.6 \\
\hline Female & 525 & 89.4 \\
\hline \multicolumn{3}{|l|}{ Working Years } \\
\hline 5 Years and Below & 161 & 27.4 \\
\hline 6-10 Years & 165 & 28.1 \\
\hline $11-15$ Years & 110 & 18.7 \\
\hline $16-20$ Years & 59 & 10.1 \\
\hline 21 Years and Above & 92 & 15.7 \\
\hline \multicolumn{3}{|l|}{ The Number of Night Shifts } \\
\hline 4 Times and Below & 318 & 54.2 \\
\hline 5-8 Times & 236 & 40.2 \\
\hline 9 Times and Above & 33 & 5.6 \\
\hline
\end{tabular}

\subsection{Variable Scores under Different Conditions}

The scores of error management climate of different genders have significant statistical significance, and the psychological flexibility and professional stress of working years have significant statistical significance. After a pairwise comparison, the error management climate score of female nurses is higher than that of male 
nurses; the occupational stress score of nurses who have worked for 11-15 years is generally higher than that of other nurses in the same group; the psychological flexibility scores of nurses working for 5 years or less and 16-20 years were higher than those of other types of nurses in the same group, as shown in Table 2.

Table2. Variable Scores under Different Conditions (Total

\begin{tabular}{cccc}
\multicolumn{4}{c}{ score \pm SD) } \\
\hline Projects & PF & EMC & OS \\
\hline Gender & & & \\
Male & $30.84 \pm 4.36$ & $52.10 \pm 7.78$ & $9.37 \pm 2.97$ \\
Female & $30.80 \pm 4.28$ & $54.39 \pm 6.39$ & $9.58 \pm 2.98$ \\
t & 0.004 & 6.809 & 0.276 \\
P & 0.952 & 0.009 & 0.600 \\
Working Years & & & \\
5 Years and Below & $31.41 \pm 3.76$ & $54.29 \pm 6.81$ & $9.12 \pm 3.08$ \\
6-10 Years & $30.28 \pm 4.67$ & $53.97 \pm 7.21$ & $9.75 \pm 2.91$ \\
11-15 Years & $30.44 \pm 4.44$ & $54.45 \pm 5.74$ & $10.16 \pm 2.82$ \\
16-20 Years & $31.90 \pm 4.06$ & $55.73 \pm 5.74$ & $8.83 \pm 2.87$ \\
21 Years and Above & $30.45 \pm 4.23$ & $52.87 \pm 6.64$ & $9.72 \pm 3.02$ \\
F & 2.781 & 1.832 & 3.165 \\
P & 0.026 & 0.121 & 0.014 \\
\hline
\end{tabular}

Table3. Descriptive Statistics

\begin{tabular}{|c|c|c|c|c|c|c|c|c|}
\hline Projects & M & SD & Gender & Working Years & The Number of Night Shifts & PF & EMC & OS \\
\hline Gender & 1.32 & 0.47 & 1 & & & & & \\
\hline Working Years & 2.58 & 1.39 & $-0.16^{* * *}$ & 1 & & & & \\
\hline The Number of Night Shifts & 1.51 & 0.60 & -0.06 & $-0.41^{* * *}$ & 1 & & & \\
\hline $\mathrm{PF}$ & 4.51 & 0.56 & -0.003 & -0.03 & -0.01 & 1 & & \\
\hline EMC & 4.51 & 0.55 & $0.11^{* *}$ & -0.03 & 0.04 & $0.60^{* * *}$ & 1 & \\
\hline OS & 1.91 & 0.59 & 0.02 & 0.04 & 0.02 & $-0.70^{* * *}$ & $-0.61^{* * *}$ & 1 \\
\hline
\end{tabular}

Note: Gender: $1=$ male, $2=$ female; Working Years: $1=5$ years and below, $2=6-10$ years, $3=11-15$ years; $4=16-20$ years, $5=21$ years and above; The Number of Night Shifts: $1=4$ Times and Below, $2=5-8$ Times, $3=9$ Times and Above. *for $\mathrm{P}<0.05, * *$ for $\mathrm{P}<0.01, * * *$ for $\mathrm{P}<0.001$, the same below.

\subsection{Regression Analysis}

First, examine the direct effect of nurses' psychological flexibility on occupational stress. It can be seen from Table 4 that psychological flexibility negatively affects occupational stress $(\beta=-0.70, \mathrm{P}<0.001)$. Second, examine the intermediary role of error management climate. It can be seen from Table 4 that psychological flexibility positively affects the error management climate $(\beta=0.60$, $\mathrm{P}<0.001)$. When the intermediary variable of error management climate is added, the negative impact of psychological flexibility on occupational pressure is weakened $(\beta=-0.52, \mathrm{P}<0.001)$, and the negative impact of the error management climate on occupational stress is still significant $(\beta=-0.30, \mathrm{P}<0.001)$, which shows that the error management climate plays a partial intermediary role between psychological flexibility and occupational stress.

\subsection{Descriptive Statistics}

First, psychological flexibility is positively correlated with error management climate $(\mathrm{r}=0.60, \mathrm{P}<0.001)$, and negatively correlated with occupational stress $(\mathrm{r}=-0.70, \mathrm{P}$ $<0.001$ ), and error management climate is negatively correlated with occupational stress $(\mathrm{r}=-0.61, \mathrm{P}<0.001)$. Second, from the perspective of demographic variables, gender is positively correlated with error management climate $(\mathrm{r}=0.11, \mathrm{P}<0.01)$, and negatively correlated with working years $(\mathrm{r}=-0.16, \mathrm{P}<0.001)$; working years are inversely related to the number of night shifts per month $(\mathrm{r}=-0.41, \mathrm{P}<0.001)$. The above conclusions provide basic data support for subsequent analysis.

Table4. The Mediating Role of Error Management Climate

\begin{tabular}{|c|c|c|c|c|c|c|c|c|c|}
\hline \multirow{2}{*}{ Predictors } & \multicolumn{3}{|c|}{ Outcome variable: OS } & \multicolumn{3}{|c|}{ Outcome variable: EMC } & \multicolumn{3}{|c|}{ Outcome variable: OS } \\
\hline & $\beta$ & $t$ & $95 \% C I$ & $\beta$ & $t$ & $95 \% C I$ & $\beta$ & $t$ & $95 \% C I$ \\
\hline Gender & 0.03 & 0.89 & {$[-0.31,0.83]$} & 0.12 & $3.53^{* * *}$ & {$[1.12,3.93]$} & 0.06 & $2.15^{*}$ & {$[0.05,1.14]$} \\
\hline Working Years & 0.03 & 0.98 & {$[-0.07,0.21]$} & 0.04 & 0.96 & {$[-0.18,0.51]$} & 0.04 & 1.37 & {$[-0.04,0.22]$} \\
\hline The Number of Night Shifts & 0.03 & 0.83 & {$[-0.18,0.45]$} & 0.07 & 1.80 & {$[-0.06,1.49]$} & 0.05 & 1.51 & {$[-0.07,0.53]$} \\
\hline $\mathrm{PF}$ & -0.70 & $-23.89^{* * *}$ & {$[-0.53,-0.45]$} & 0.60 & $18.44^{* * *}$ & {$[0.83,1.03]$} & -0.52 & $-14.97^{* * *}$ & {$[-0.41,-0.32]$} \\
\hline EMC & & & & & & & -0.30 & $-8.45^{* * *}$ & {$[-0.17,-0.10]$} \\
\hline $\mathrm{R}$ & \multicolumn{3}{|c|}{0.71} & \multicolumn{3}{|c|}{0.61} & \multicolumn{3}{|c|}{0.74} \\
\hline $\mathrm{R}^{2}$ & \multicolumn{3}{|c|}{0.50} & \multicolumn{3}{|c|}{0.38} & \multicolumn{3}{|c|}{0.55} \\
\hline $\mathrm{F}$ & \multicolumn{3}{|c|}{$143.869^{* * *}$} & \multicolumn{3}{|c|}{$88.183^{* * *}$} & \multicolumn{3}{|c|}{$143.313^{* * *}$} \\
\hline
\end{tabular}

Table5. The intermediary role of all dimensions of error management climate

\begin{tabular}{|c|c|c|c|c|c|c|c|c|c|}
\hline \multirow{2}{*}{ Predictors } & \multicolumn{3}{|c|}{ Outcome variable: ETL } & \multicolumn{3}{|c|}{ Outcome variable: EC } & \multicolumn{3}{|c|}{ Outcome variable: ECF } \\
\hline & $\beta$ & $t$ & $95 \% C I$ & $\beta$ & $t$ & $95 \% C I$ & $\beta$ & $t$ & $95 \% C I$ \\
\hline Gender & 0.09 & $2.63^{* *}$ & {$[0.26,1.77]$} & 0.12 & $3.39^{* *}$ & {$[0.32,1.19]$} & 0.13 & $3.61^{* * *}$ & {$[0.35,1.18]$} \\
\hline Working Years & 0.04 & 1.00 & {$[-0.09,0.28]$} & 0.01 & 0.28 & {$[-0.09,0.12]$} & 0.04 & 1.13 & {$[-0.04,0.16]$} \\
\hline The Number of Night Shifts & 0.06 & 1.46 & {$[-0.11,0.73]$} & 0.06 & 1.46 & {$[-0.06,0.42]$} & 0.07 & 1.92 & {$[-0.01,0.45]$} \\
\hline PF & 0.55 & $16.14^{* * *}$ & {$[0.38 .0 .49]$} & 0.54 & $15.50^{* * *}$ & {$[0.21,0.27]$} & 0.57 & $16.82^{* * *}$ & {$[0.22,0.28]$} \\
\hline
\end{tabular}




\begin{tabular}{|c|c|c|c|c|c|c|c|c|c|}
\hline $\begin{array}{c}\mathrm{ECF} \\
\mathrm{R} \\
\mathrm{R}^{2} \\
\mathrm{~F}\end{array}$ & & $\begin{array}{r}0.5 \\
0.3 \\
66.82\end{array}$ & & & $\begin{array}{r}0 . \\
0 . \\
63.2\end{array}$ & & & $\begin{array}{r}0.5 \\
0.3 \\
74.07\end{array}$ & \\
\hline Table5. & ontinu & 1). The in & rmediary role & f all $\mathrm{c}$. & nensions & f error manag & nent c & mate & \\
\hline \multirow{2}{*}{ Predictors } & \multicolumn{3}{|c|}{ Outcome variable: OS } & \multicolumn{3}{|c|}{ Outcome variable: OS } & \multicolumn{3}{|c|}{ Outcome variable: OS } \\
\hline & $\beta$ & $t$ & $95 \% C I$ & $\beta$ & $t$ & $95 \% C I$ & $\beta$ & $t$ & $95 \% C I$ \\
\hline Gender & 0.06 & 1.95 & {$[-0.003,1.07]$} & 0.05 & 1.68 & {$[-0.08,1.04]$} & 0.05 & 1.73 & {$[-0.07,1.06]$} \\
\hline Working Years & 0.04 & 1.43 & {$[-0.04,0.22]$} & 0.03 & 1.07 & {$[-0.06,0.21]$} & 0.04 & 1.27 & {$[-0.05,0.22]$} \\
\hline The Number of Night Shifts & 0.04 & 1.45 & {$[-0.08,0.51]$} & 0.04 & 1.19 & {$[-0.12,0.49]$} & 0.04 & 1.29 & {$[-0.11,0.51]$} \\
\hline $\mathrm{PF}$ & $-\overline{-}$ & $16.09^{* * *}$ & {$[-0.33 .-0.22]$} & $\begin{array}{c}- \\
0.60\end{array}$ & $17.59^{* * *}$ & {$[-0.46,-0.37]$} & $\overline{-}-\overline{59}$ & $16 . \overline{9} 0^{* * *}$ & {$[-0.46,-0.36]$} \\
\hline ETL & $\overline{-}$ & $-9.32^{* * *}$ & & & & & & & \\
\hline EC & & & & $0 . \overline{19}$ & $-5.61^{* * *}$ & {$[-0.40,-0.19]$} & & & \\
\hline ECF & & & & & & & $\overline{-}-$ & $-5.60^{* * *}$ & {$[-0.42,-0.20]$} \\
\hline $\mathrm{R}$ & \multicolumn{3}{|c|}{0.75} & \multicolumn{3}{|c|}{0.72} & \multicolumn{3}{|c|}{0.72} \\
\hline $\mathrm{R}^{2}$ & \multirow{2}{*}{\multicolumn{3}{|c|}{$\begin{array}{c}0.56 \\
149.453^{* * *}\end{array}$}} & \multirow{2}{*}{\multicolumn{3}{|c|}{$127.427^{* * *}$}} & \multirow{2}{*}{\multicolumn{3}{|c|}{$127.390^{* * *}$}} \\
\hline $\mathrm{F}$ & & & & & & & & & \\
\hline
\end{tabular}

\section{Discussion}

\subsection{The status quo of psychological flexibility, error management climate and occupational stress}

This study found that nurses' psychological flexibility and hospital error management climate are good, while occupational stress is at the lower middle level. This shows that the psychological flexibility of nurses and the level of hospital error management climate have a better effect on relieving the occupational stress of nurses. The conclusion of this study well confirms the research conclusion that psychological flexibility can better predict negative emotions and well-being ${ }^{[13]}$. Work requirementsresource model believes that when individuals feel stimuli from outside, for example, when they feel greater pressure from work, they will choose to use their existing resources to make up and relieve the discomfort caused by these external stimuli, these resources include both external and internal, including both physical and spiritual resources. Relevant research shows that when the above-mentioned resources are lacking, it will increase the difficulty for individuals to complete their work, and then cause various adverse consequences for individuals. On the contrary, if the individual has enough working resources, it will inhibit the production of these adverse consequences. Psychological flexibility represents one of the internal work resources mentioned above. As a nurse, you should have a high degree of psychological flexibility to deal with various stresses during the outbreak and avoid medical and nursing accidents caused by occupational stress.

At the same time, it is inevitable to make mistakes in the work. What is important is how to manage and correct the mistakes. According to social information processing theory, individual's behavior will be affected by external environment, and individual's perception and judgment of external environment will also affect individual's behavior ${ }^{[14]}$. Error management climate refers to the practice, attitude, cognition or opinion of individuals on error management of their organizations, including both positive error management climate and negative error management climate ${ }^{[15]}$. In this study, it belongs to a positive error management climate, and this study shows that when nurses make mistakes in their work, they don't choose to hide or keep silent for self-protection and other reasons. Instead, they learn from the mistakes, communicate with them and prevent similar mistakes in future work ${ }^{[16-17]}$. During the outbreak of the epidemic, these practices are worthy of the majority of nurses to follow and learn.

\subsection{The influencing factors of psychological flexibility, error management climate and occupational stress}

Gender may be the influencing factor of the error management climate, and working years may be the influencing factor of psychological flexibility and occupational stress. When errors occur, compared with men, women are more likely to lay down their body and face, communicate, communicate and learn with colleagues, learn in the errors, and prevent similar errors in the future work. However, due to their face and selfesteem, men tend to treat the errors negatively, which leads to the higher score of error management climate of female nurses than men. In terms of psychological flexibility, nurses who have worked for a long time tend to accumulate a lot of work experience, both psychological level and nursing technology level are very high, so when they encounter various problems, they can use their own resources and advantages to solve problems quickly. But for the nurses who just entered the workplace, although their own experience and accumulation is not as much as their predecessors, they need to quickly improve their own work requirements and conditions, so that they can be used at any time in the work.

\subsection{The intermediary role of hospital error management climate}


Another finding of this study is that error management climate and its dimensions play a part of intermediary role between psychological flexibility and occupational stress. In practice, nurses can avoid the impact of occupational stress by improving their psychological flexibility. At the same time, the hospital to create a good, open, positive error management climate can also offset the adverse effects of occupational stress. This conclusion is similar to that of Zhang and others, and there is a negative correlation between psychological flexibility and stress level ${ }^{[18]}$. When the level of psychological flexibility is high, the degree of empirical avoidance is low, which shows that when individuals encounter external stimulation, they do not directly respond to the existing experience, but choose to mobilize all kinds of work resources, reasonably choose all kinds of resources to actively respond to external stimulation, and further offset the negative consequences caused by external stimulation. At the same time, Hodges and Gardner also confirmed in the study that error management climate negatively affects work stress ${ }^{[19]}$, which is consistent with the conclusion of this study. In a high-level error management climate, individual parallax error is an opportunity to learn and improve, not too much anxiety and pressure to make mistakes, but actively discuss errors. These characteristics are conducive to reducing occupational stress and improving mental health level ${ }^{[20]}$. Therefore, according to the recommendations of this study, in order to reduce the occupational stress of nurses during the epidemic or in the future major public health emergencies, the following measures can be taken: (1) improve the psychological flexibility of nurses. To increase the training of nurses' mental health and working skills, we can adopt expert lectures, online training, outdoor team training and other ways, so that they can master all kinds of working conditions necessary for the nursing industry, so that they can be proficient in the future work; (2) create an open and good error management climate. On the one hand, the hospital needs to establish a reasonable reward and punishment system and incentive measures. For some errors, nurses are encouraged to exchange and learn among members, so as to make up for the loss of work caused by the errors as soon as possible. On the other hand, the hospital can regularly carry out knowledge propaganda lectures to encourage the nurses who make mistakes and correct to share their experiences and learn together.

\subsection{Limitations and future prospects}

There are some limitations in this study: (1) the results of this study show that gender has no statistical significance in the impact of error management climate, and working years have no statistical significance in the impact of psychological flexibility and occupational stress, which may be related to the small sample size within the group and the unequal sample size between groups. In the future, we need to further expand the scope of data collection, and further increase the sample size for verification; (2) in terms of research methods, psychological flexibility belongs to the variable of individual level, and error management climate belongs to the variable of group level.
Therefore, cross level empirical analysis method can be used in the future, taking into account the influence of group level and individual level on pressure perception.

\section{5 conclusion}

This study explored the influence of psychological flexibility on occupational stress and the mediating effect of error management climate in nurses during the COVID2019 under the background of big data. The results show that error management climate and its dimensions have a partial intermediary effect between psychological flexibility and occupational stress, and both psychological flexibility and error management climate have a negative impact on occupational stress. The level of psychological flexibility and error management climate of nurses is relatively high, and the occupational stress is in the lower middle level; gender may be the influencing factor of error management climate perceived by nurses, and working years may be the influencing factor of psychological flexibility and occupational stress. In summary, during the new coronary pneumonia epidemic and major public health emergencies under the background of big data, hospital managers can reduce occupational pressure by improving nurses' psychological flexibility and improving the error management climate, so as to maintain the sustainability and stability of nursing work and team, and improve the overall nursing level and hospital medical level.

\section{References}

1. X. L. Liu, Y. S. Cheng, M. Y. Wang, Y. Pan, H. Guo, R. H. Jiang et al., "Psychological state of nursing staff in a large scale of general hospital during COVID-19 epidemic," Chin J Nosocomiol, vol.30, pp.1634-1630, March 2020.

2. P. Chen, Q. D. Sun, W. Chen, H. Wang, X. E. Chen, C. H. Zhao et al., "Countermeasures for prevention and control of infection in patients and medical staff of specialized hospital of COVID-19," Chin J Nosocomiol, vol.30, pp.1-5, March 2020.

3. X. J. Lao, Y. Chen, W. Y. Shao, "Disaster Preparedness and Core Competency of Disasters Rescue of Emergency Nurses in Quzhou City," Medicine and Society, vol.31, pp.41-43, Oct. 2018.

4. L. Jiang, Y. Kong, T. T. Yin, "Research progress of nurses' psychological quality in disaster relief in China," Nurs J Chin PLA, vol.29, pp.35.37,June 2012.

5. S. Hayes, J. B. Luoma, F. W. Bond, A. Masuda, J. Lillis, "Acceptance and commitment therapy: model, processes and outcomes," Behav Res Ther, vol.56, pp.1-2, Jan. 2006.

6. C. Zhang, H. Cao, Z. W. Huang, Z. R. Huang, H. H. Liu, "Relation of psychological flexibility to negative affectivity and happiness in college students," Chinese Mental Health Journal, vol.33, pp.868.874, Oct. 2019.

7. C. M. Wang, Z. Q. Hong, "Linking Error 
Management Climate to Organizational Effectiveness: An Empirical Evidence," Journal of Zhejiang University(Humanities and Social Sciences), vol.30, pp.114-119, Oct. 2000.

8. J. Cao, Y. Ji, Z. H. Zhu, "Reliability and validity of the Chinese version of the Acceptance and Action Questionnaire-Second Edition (AAQ-II) in college students," Chinese Mental Health Journal, vol.27,pp.873-877, Nov. 2013.

9. N. J. Hulbert williams, L. Storey, "Psychological flexibility correlates with patient-reported outcomes independent of clinical or socio-demographic characteristics," Support Care Cancer, vol.24, pp.1-9, June 2016.

10. Z. Y. He, "Research on the Relationship between Error Management Climate and Service Innovation Behavior of Employee in Exhibition Enterprise - - Organizational Identification as the Intermediary Variable," Guangzhou: South China University of Technology, April 2016.

11. C. Van Dyck, M. Frese, M. Baer, S. Sonnentag, "Organizational error management cultureand its impact on performance: A two study replication," Journal of Applied Psychology, vol.90, pp.1228-1240, 2005.

12. J. Cao, "Study on the Relationship between Job Stressors and Job Bournout \& its Influential Factors of Managers," Shanghai: Tongji University, Dec. 2005.

13. E. J. Marshall, R. N. Brockman, "The relationships between psychological flexibility, self-compassion, and emotional well-being," J Cog Psychotherapy, vol.30, pp.60-72, Jan. 2016.

14. H. Ding, X. Q. Lin, Y. Y. Xu, "Mechanism Research on Effect of Strengths-based Psychological Climate on Employee Innovative Behavior," Nankai Business Review, vol.21, pp.28-38, Feb. 2018.

15. Y. Li, S. M. Wu, "Impact of Error Management Climate on Employees Innovation: The Moderating Effect of Paternalistic Leadership," Science and Technology Management Research, pp.149-158, Feb. 2019.

16. Y. Q. Xie, Y. Li, W. T. Wang, "Discussions on Error Reporting in Organizations," East China Economic Management, vol.29, pp.162-167, Jan. 2015.

17. Y. Li, Y. Q. Xie, X. Fang, "Research on Error Management Culture in the Perspective of Error Information," Journal of Modern Information, vol.33, pp. 19-22+48, June 2013.

18. L. N. Zhang, C. J. Chu, "Relationship Among StateTrait Anxiety, Perceived Stress, Coping Styles and Psychological," Medicine and Society, vol.31, pp.6769+73, Oct. 2018.

Flexibility of Medical Graduate Students in a University in Dongguan City

19. M. E. Hodges, D. Gardner, "Examining the influence of error climate on aviation maintenance performance," Australasian Journal of Organisational
Psychology, vol.7, pp.e4, Feb. 2014.

20. K. Yin, J. M. Sun, L. N. Chen, "Error Management Climate: A Literature Review and Prospects," Foreign Economices \& Management, vol.38, pp.75-87, Feb, 2016. 